\title{
The Application of Distance learning Engineering Mechanism in New Generation Migration Workers
}

\author{
Haifeng Tian ${ }^{1, a}$, Zezhao Liü,b \\ ${ }^{1}$ Dept. of Business Management, Shaanxi Radio \& TV University, Xi an, China \\ ${ }^{2}$ Dept. of Business, Shaanxi Radio \& TV University, Xi`an, P.R.China \\ apreliu@eyou.com, bHaiffengweifu@163.com
}

Keywords: Continuing education, New generation farmer; E-learning, Western China.

\begin{abstract}
The study aims to investigate the feasibility of e-learning application as an alternative way to conduct continuing education (CE) for New Generation Migrate Workers (NGMW) on the context of western China. A enterprise-based cross-sectional survey was conducted with a randomly selected sample of 183 NGMWs in Xi'an city, Shaanxi Province. A structured questionnaire was used to collect data. Results reveals that NGMWs who participated in e-learning education experiences have divergences in groups Reasons for adopting e-learning included achieving life learning, fulfilling personal interests, job needs, information diversity, etc. Objects who rejected e-learning as their way of CE include poor computer competence, lack of software condition, heavy work load, conflict with personal preference, heavy economic burden, lack of motivation, and low self-control. Reasons were analyzed eventually to provide directions for decreasing barriers for developing e-learning for NGMWs in western China.
\end{abstract}

\section{Introduction}

When new generation of the peasant workers face career choice there will be more challenges, as also affects their education desires. Little attention has been paid, however, to the continuing education situation of a new working class: more than 100 million peasant-workers aged on average16-28, who have streamed from the countryside to the cities over the past decade (Perry, 1993; Walder, 1984) [1]. When we refer to the new generation of dagongmei/zai, we mean those people who were born in the late 1980s and 1990s, and who grew up in either urban areas or rural communities. The new generation of migrant workers (NGMW) has been defined by its "structure of feeling" and its ways of life. Characteristic of the new generation's ways of life is a greater disposition toward individualism, an increased proclivity for urban consumer culture (Davis, 2000; Pun, 2003; Yan, 2008) [2-4], less constrained economic circumstances and greater pursuit of personal development and freedom (Jacka, 2006) [5], a higher rate of job turnover and less loyalty to their work (Smith et al., 2004), and a greater level of spontaneous collective actions at the workplace (Chan and Pun, 2009) [6-7].

According to the Long run Education Plan (2010-2020) of People's Republic of China, "Continuing education involves: participation in professional and scientific programs and seminars, courses and other continuing education programs". E-learning program (or web-based on-line learning) involves learning activities, which is carried out with digital tools and access to teaching materials through wired and wireless networks. In an era of knowledge economy, the Internet is expected to play an important part in providing continuing education (CE) for NGMWs. E-learning, as a diverse learning style, has been widely recognized in several countries and has become a legitimate learning method for youth in the new century (DiMaria-Ghalili et al., 2005) [8]. The data shows that in nearly ten years globally the number of online registration for the ratio of students in higher education has been up to more than 25\%. Survey of MIT reveals that in 2010 USA enrollment students for on-line learning also elevated to more than one million. Among this trend, groups of young people occupy a large proportion of e-learning services. On the whole, studies based on western context has extended for long width, while research in the application of web-based learning 
in providing CE for NGMWs in China is still in its early stage. Whether and why NGMWs adopt or reject e-learning as new way of CE still needs to be confirmed. Therefore, the main purposes of this study are to investigate the feasibility of e-learning for establishing NGMWs continuing education and to further examine the reasons for adopting or rejecting e-learning as their alternative way of continuing education [9].

\section{Method \& Procedure}

The general research framework of this study adopted a problem-based research cycle presented by Levy, et al. To achieve the study goals described in the Introduction, this study addresses the indicated research questions and employs both qualitative and quantitative data analyses to produce and explore new findings. Qualitative analysis and literature review is conducted to support and validate all of the issues.

Research participants. The study was a descriptive with prospective approach involving the surveys of NGMWs employed in enterprises of Xi'an city, Shaanxi Province. A cluster random-sampling method was used to select sample data. All were stratified into three strata: large-sized, medium-sized, micro-firm. Then, enterprises in each stratum were proportionally selected by a cluster random sampling; NGMWs working at the selected enterprise comprised the study sample [10-13]. Data were collected by e-mailing the questionnaire. At the end, of 361 recipients 183 returned completed questionnaires, with a return rate of $72.9 \%$. Using a $\mathrm{x} 2$ test, no significant differences were seen in PHN proportion in three levels $(\mathrm{X} 2=1.76, \mathrm{P}>0.05)$; i.e., the sample was representative of the population.

Instruments. E-learning Continuing Education Analysis Questionnaire was developed to provide information about the NGMWs personal desires. It consisted of close and open questions about specified topics in e-learning. On the whole, it included two main parts: Independent variables: age, education level, marital status, job position, types of enterprises involved, and previous experience in web-based learning. Dependent variables: this category included two parts, the feasibility of adopting e-learning as an alternative way of continuing education, and reasons for adopting or rejecting e-learning.

- Feasibility of adopting e-learning. A four-point question was used to examine NGMWs' perceived likelihood of adopting e-learning as their continuing education method in the future. "'Definitely possible", " possible", " not possible", "'definitely not possible" corresponded to points 4, 3, 2, and 1, respectively. Scores greater than and equal to 3 meant affirmative possibility of adopting e-learning; conversely, scores less than and equal to 2 meant negative possibility (i.e., rejection).

- Reasons for adopting or rejecting e-learning. A structured questionnaire, called Reasons for Adopting or Rejecting E-learning Scale, was used to examine the reasons why NGMWs adopt or reject e-learning. This scale was a 20 -item self-reporting scale. It included two separate dimensions: the reasons for adopting (11 items) and the reasons for rejecting (9 items). For single items, a yes/no question was used to collect data; one point was given to "yes" answers, and zero points were given for "no" answers.

- validity \& reliability. To evaluate questionnaire validity and reliability, three experts in the areas of distance education or youth education checked the questionnaire's content validity. Four NGMWs completed the face validity test. Regarding reliability of this study, the agreement of behavioral intention toward e-learning (a four-point question) was $80 \%$.

Data analysis. Data collected in this survey were processed electronically. Of the 260 questionnaires dispersed to the NGMWs, 78\% were adequately completed and processed. The data were analyzed using the SPSS 19.0.and expressed as median value (with SDs) or numbers (with percentages). Mann-Whitney U-tests were used for comparison between groups. Significance level was taken at $p<0.05$. Frequency and percentage were used to describe subjects' backgrounds as well as to investigate the feasibility of developing e-learning and to examine the reasons for adopting or rejecting e-learning. 


\section{Results}

Basic data of the subjects. The mean age of the participating subjects was 19.83 years ( $\mathrm{SD}=$ 6.19). 79.8\% had graduated from junior education institution. Most subjects were not married $(91.03 \%)$. Over one-half $(67.22 \%, \mathrm{n}=183)$ of the subjects worked in small sized enterprises. Participants' locations in medium and large enterprises type were $23.07 \%$, and $8.03 \%$ respectively. Only $23.88 \%$ of the subjects had ever participated in an e-learning program .For the Feasibility of e-learning, most of the subjects (73.29\%) replied that it was "highly possible" or "possible" for them to take continuing education programs via e-learning in the future . However, $11.3 \%$ subjects rejected e-learning as their alternative way of continuing education(Tables 1).Additionally, there were significant differences among NGMWs in interest in continuing education in relation to the enterprises affiliated( $\mathrm{F}=231.94 ; \mathrm{p}<0.001)$, namely, youths from large enterprises were more interested in continuing education(See Table 1).

B. Reasons for adopting or rejecting e-learning

Among a total of 183 NGMWs, more than one-half of subjects expressed that e-learning was beneficial for achieving lifelong learning (61.39\%), especially because of allowing them to choose curriculum and content based on personal learning needs (See Tables 2).

In the adopting group $(n=149)$, who tended to adopt e-learning as the alternative way of continuing education, more than one-half of subjects expressed that e-learning was beneficial for them to achieve lifelong learning $(60.87 \%)$. The five main reasons given for adopting e-learning included: selecting curriculum and content based on personal learning needs, broadening one's horizon and/or learning the newest knowledge, time-savings, and information diversity. In contrast, the rejecting group $(n=34)$ indicated that poor computer competence, lack of adequate computer facilities, time conflict or unmatched interests existing between job and education content and heavy family burdens were the five main reasons for rejecting.

Table 1. Basic data of the surveyed NGMW subjects $(n=183)$

\begin{tabular}{|l|c|c|}
\hline \multicolumn{1}{|c|}{ Variables } & $\mathrm{n}$ & $\%$ of the total ( $\mathrm{n}=183)$ \\
\hline Educational level & & \\
\hline Junior school & 11 & 6.01 \\
\hline Middle school & 72 & 86.7 \\
\hline Vocational senior high school & 31 & 16.9 \\
\hline Junior college or above & 69 & 37.7 \\
\hline Marital status & & \\
\hline Not married & 162 & 88.5 \\
\hline Married & 21 & 11.48 \\
\hline Job position type & & \\
\hline Large state-owned enterprise & 7 & 3.82 \\
\hline Middle--sized enterprise & 76 & 41.5 \\
\hline Small-sized enterprise & 100 & 54.6 \\
\hline Previous experience in web-based learning & & \\
\hline Yes & 112 & 61.2 \\
\hline No & 71 & 38.8 \\
\hline Adopt e-learning & & \\
\hline Yes & 101 & 55.1 \\
\hline No & 82 & 44.8 \\
\hline
\end{tabular}

Table 2. Main reasons for NGMWs adopting e-learning programs $(n=183)$

For some NGMWs, not having a home computer and being without an internet access are their stated barriers to adopting e-learning, for economic burden might impede them from learning at home. Thus, there is a continuing need for government support to enable more people to internet access. Government strategies for training NGMWs in basic computer competence will help ensure internet access at worksites, promising NGMWs continuing education via e-learning and increasing incentives for learning (e.g. an official or academic certification). These steps will make development of e-learning for NGMWs both feasible and successful. Generally, Administrative support is critical in developing e-learning programs and in building supportive environments that motivate NGMWs' participation in e-learning programs in western rural China.

\section{Findings \& Summary}

This finding confirmed that e-learning (via the Internet) is a highly feasible way and worthy of development as a means of NGMWs' continuing education. Most NGMWs believe e-learning could 
fulfill their personal learning needs and job demands. Learning needs assessment is critical before developing an e-learning course that is capable of fulfilling personal needs and job demands simultaneously. However, providing diverse learning programs might be more feasible for fulfilling various needs. According to this finding, we emphasize three principles (diversity, individualization, and task-orientation) that should be specifically considered. It is also necessary to increase search information ability and to provide clear e-learning information in order to help NGMWs acquire desirable and effective e-learning programs. For some NGMWs, self-controlling ability might be one consideration for adopting or rejecting e-learning. For individuals with less self-control, it is highly important to establish user-friendly learning environment on the Internet in the beginning stages of development.

Apart from hardware and software reasons, heavy workload, job stress and family duties all might impede NGMWs' adoption of e-learning, or make a negative impact on NGF continuing education. Therefore, education policy makers should consider how to increase NGMWs' motivation and to improve NGMWs' time-management skills so that they may be able to participate in e-learning and achieve improved educational outcomes. E-learning is a feasible and valuable learning model that is worth making generally available to NGMWs. From analyses findings, we have confirmed that asynchronous e-learning programs are a suitable method for NGMW continuing education, especially for individuals with high self-control that allows NGMWs to learn at remote locations according to their own needs and pace. Needs assessment is strongly recommended in the program preparation stage. Only by fulfilling NGMWs' individual needs (personal and job) can this new e-learning style be successful. Multi-channel learning patterns are still considered for inclusion in NGMW continuing education because not all NGMWs are accustomed to e-learning. Furthermore, regarding the effectiveness of e-learning programs, we suggest that further studies are required.

The findings of this study offer direction to planners of continuing education programs for NGMWs in western China. Different forms of continuing education should be used in order to enable NGMWs to obtain demanded points without any pressure and maintain their professional practice in a rapidly changing world. Further research should be focused on proving benefits of professional education and improvement with particular emphasize on quality of NGMW's benefit.।

\section{Discussion}

This investigation resulted from an increased attention dedicated to continuing education in Western China perspective. In this study we identified the current status of e-learning upon continuing education and interest of NGMWs. We attempted to identify the most appropriate form and method of e-learning education which will satisfy the educational needs of NGMWs.

The findings of this study offer direction to planners of continuing education programs for NGMWs in China, especially for the western geographical regions. Also, based on these findings, public supportive continuing education programs should be planned and organized by local educational administrative organs yearly with the topics proposed by themselves, taking into account their specialty and urgent concerns, for it increases their perception in acceptance. Different forms of continuing education should be applied in order to enable NGMWs to obtain legally demanded points for credentials upheaval without over economic pressure and maintain their professional practice in a rapidly changing world. Further research should be focused on proving benefits of professional education and improvement with particular emphasize on quality of educational benefits. In addition, this questionnaire does not offer analysis of innate continuing education mode of NGMWs for e-learning, which is one of basic limitations in this paper.

\section{Acknowledgment}

This research was financially supported by the National Twelve-Five Education Science Planning of P.R.China (Grant No. FJB110131) . We would like to express our great appreciation to Professor G.Hu, Jiangsu Normal University, for his professional and unselfish guidance and support throughout 
this research. Also, we would like to thank our students from Shaanxi Business College for their support and contribution during the writing of the paper, particularly with data collection. Our deepest appreciation and gratitude goes to those volunteers for their kind participation in the questionnaire survey, allowing us to collect valuable data and produce this paper.

\section{References}

[1] Walder, Andren. The remaking of the Chinese working class, 1949-1981. Modern China. 10(1984)3-48.

[2] Davis, Deborah.The Consumer Revolution in Urban China. Univ. of California Press. Berkeley, 2000.

[3] Pun. Ngai. Sub-sumption or consumption? The phantom of consumer revolution in globalizing China. Cultural Anthropology.18(2003)469-492.

[4] Yan Hairong. New Masters, New Servants: Migration, Development and Women Workers in China. Durham, NC: Duke Univ. Press.2008.

[5] Jacka, Tamara. Rural Women in Urban China: Gender, Migration and Social Change. Armonk, NY: M.E. Sharpe.2006.

[6] Smith, Chris, M. Daskalaki, T. Elger, and D. Brown. Labor turnover and management retention strategies in new manufacturing plants. International J. of Human Resource Management. 15, (2004)371-96.

[7] Chan, K. Chi and P. Ngai. The making of a new working class: a study of collective actions of migrant workers in south China. China Quarterly. 198 (2009)287-303.

[8] DiMaria-Ghalili, R.A., Ostrow, L., Rodney, K., Webcasting: a new instructional technology in distance graduate nursing education. Journal of Nursing Education. 44 (2005)11-18.

[9] S. Yu and K.F. Yang, Attitudes towards web-based distance learning among public health nurses in Taiwan: a questionnaire survey. International Journal of Nursing Studies. 43 (2006)767-774.

[10] R.E. Yellen, Distance learning students: a comparison with traditional students. Journal Educational Technology Systems.26 (1998) 215-224.

[11] M.R. Garland, Student perceptions of the situational, institutional, dispositional and epistemological barriers to persistence. Distance Education 14 (1993) pp.181-198.

[12] Levy, Timothy J. Ellis and Yair. "Framework of Problem-Based Research: A Guide for Novice Researchers on the Development of a Research-Worthy Problem. Informing Science: the International Journal of an Emerging Trans-discipline. 8 (2008) 17-33.

[13] M.R. Garland, Student perceptions of the situational, institutional, dispositional and epistemological barriers to persistence. Distance Education 14 (1993)181-198. 